\section{Leishmania infection in psoriasis}

Dear Editor,

In this Journal Brunet and colleagues ${ }^{1}$ discussed reactivation of latent infections in the context of chronic disease, solid organ transplantation or long-term immunosuppressive treatment. We recently observed the reactivation of Leishmania infection in a 46-year-old patient receiving methotrexate for psoriasis, who was diagnosed with visceral leishmaniasis (VL) showing a mucocutaneous involvement. We analyzed the epidemiologic and clinical characteristics of all cases of leishmaniasis in patients with psoriasis found through a review of the literature.

Our patient was admitted into the Infectious Disease Unit of Paolo Giaccone hospital, in Palermo, with a painless and ulcerated lesion onto the oral mucosa (Fig. 1a), two nodular ulcerated lesions on the right knee and another one on instep of the right foot appeared one month before (Fig. 1b). The patient did not travel outside Italy during the last year. He had been suffering from lowgrade fever in the last month.

Considering the above findings leishmaniasis was suspected and a needle aspiration of oral and cutaneous lesions was arranged in order to perform microscopy and Leishmania-PCR, which were positive for Leishmania. Laboratory tests exhibited: WBC 4970/mmc, Hb $13.9 \mathrm{~g} / \mathrm{dl}$, C Reactive Protein, $26.9 \mathrm{mg} / \mathrm{L}$; positive serology for Leishmania (IgG 1/3200) and positive LeishmaniaPCR test on peripheral blood. Abdominal US examination revealed splenomegaly $(14 \mathrm{~cm})$; methotrexate was suspended and liposomal Amphotericin B, $4 \mathrm{mg} / \mathrm{kg}$ per day for 10 days, followed by two further administrations two weeks later was started. Cutaneous and mucosal lesions improved at the end of the first 10 days of therapy and completely vanished after two further administrations, 40 days from the beginning of treatment. Leishmania-PCR on peripheral blood after 10 days of therapy was negative.

Table 1 shows the literature data about characteristics, therapy and outcome of patients with psoriasis and leishmaniasis.

Leishmaniasis is a vector-born chronic infectious disease caused by protozoa of the genus Leishmania and transmitted to humans by the bite of phlebotomine sandflies. In Europe, the Mediterranean countries are the most affected areas. Leishmania parasite establishes chronic intracellular parasitism, survives for an infected person's lifetime and, in the event of major immune deficiency, may be reactivated from sites of latency. Leishmaniasis can present with a spectrum of clinical manifestations and three patterns of infection are described: cutaneous $(\mathrm{CL})$, mucosal or mucocutaneous (ML or $\mathrm{MCL}$ ) and visceral leishmaniasis (VL). The infecting species of Leishmania is very important in determining the clinical manifestations and the host immune response is crucial in determining the clinical outcome of infection ${ }^{2}$. Today, non-HIV related immunosuppressive conditions are becoming increasingly prevalent, mainly because of better medical care of patients with chronic
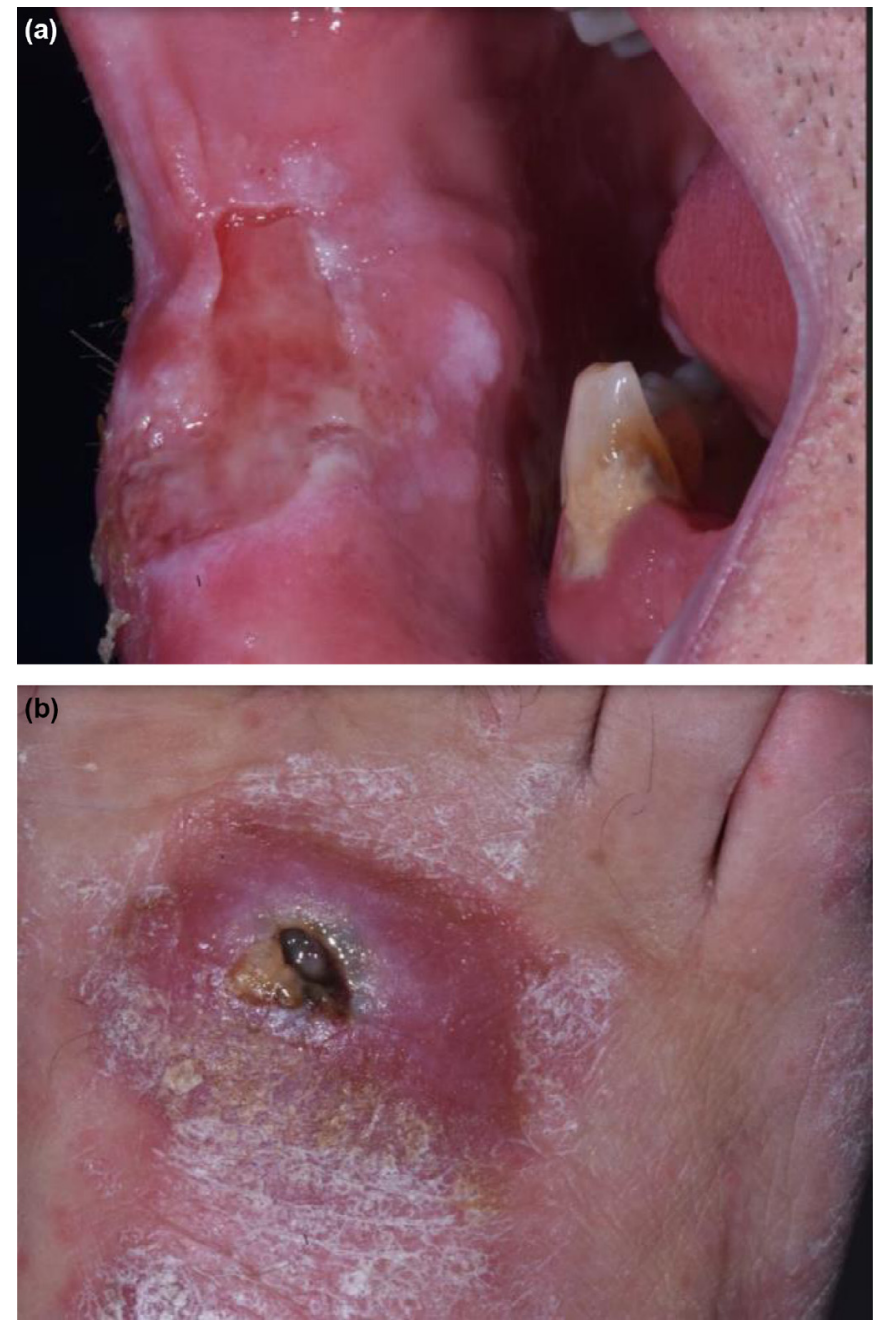

Figs. 1a and b. Ulcerated lesions by Leishmania onto the oral mucosa (a) and right foot (b) in a 46-year-old patient with psoriasis.

illnesses and the therapeutic use of immunosuppressive drugs. In the field of rheumatology, leishmaniasis has been reported in association with the use of various immunosuppressive drugs. ${ }^{3}$ The introduction of tumor necrosis factor-alpha (TNF- $\alpha$ ) antagonist drugs has received much attention recently and several cases of VL have been reported in rheumatic patients who do anti-TNF $\alpha$ drugs. 4

Psoriasis is a chronic inflammatory autoimmune disease affecting $2-3 \%$ of the world's population and characterized by an aber- 
Table 1

Detailed characteristics of patients with psoriasis or psoriatic arthritis in whom leishmaniasis developed.

\begin{tabular}{|c|c|c|c|c|c|c|c|c|c|c|c|c|}
\hline $\begin{array}{l}\text { First author Year } \\
\text { Number of patients }\end{array}$ & $\begin{array}{l}\text { Risk factors for } \\
\text { Leishmania } \\
\text { infection }\end{array}$ & Age, $y / \operatorname{sex}$ & Disease & $\begin{array}{l}\text { Immunosuppressive } \\
\text { treatment }\end{array}$ & $\begin{array}{l}\text { Duration } \\
\text { months }\end{array}$ & $\begin{array}{l}\text { Form of } \\
\text { Leishmania } \\
\text { Infection }\end{array}$ & PCR & Microscopy. & Serology & Treatment & $\begin{array}{l}\text { Suspension } \\
\text { of immuno- } \\
\text { suppressive } \\
\text { therapy }\end{array}$ & Outcome \\
\hline $\begin{array}{l}\text { Romani-Costa et al., 2004, } \\
\text { Enferm Infecc Microbiol } \\
\text { Clin }\end{array}$ & $\begin{array}{l}\text { Endemic area } \\
\text { Spain }\end{array}$ & $55 / \mathrm{M}$ & PSA & Infliximab & 9 & $\mathrm{VL}$ & & & & Meglumine antimoniate & & Cured \\
\hline $\begin{array}{l}\text { Saha et al., 2006, Br J } \\
\text { Dermatol }\end{array}$ & $\begin{array}{l}\text { Traveler returning } \\
\text { from Mexico, Spain, } \\
\text { Greece, Malta and } \\
\text { Turkey }\end{array}$ & $37 / \mathrm{M}$ & PSA & $\begin{array}{l}\text { Metotrexate } \\
\text { Sulfasalazine }\end{array}$ & 36 & $\mathrm{CL}$ & Pos SB & Pos SB & Pos & $\begin{array}{l}\text { Iv sodium } \\
\text { stibogluconate }\end{array}$ & NR & Cured \\
\hline $\begin{array}{l}\text { Balato et al., } 2008, \\
\text { Dermatology }\end{array}$ & $\begin{array}{l}\text { Endemic area } \\
\text { Italy }\end{array}$ & $42 / \mathrm{M}$ & $\mathrm{P}$ & $\begin{array}{l}\text { Efalizumab } \\
\text { cyclosporina }\end{array}$ & 24 & $\mathrm{VL}$ & $\begin{array}{l}\text { Pos BM } \\
\text { Pos } \\
\text { PB }\end{array}$ & Pos BM & Pos & None & Yes & Cured \\
\hline $\begin{array}{l}\text { Tektonidou and Skopouli, } \\
\text { 2008, Clin Rheumatol }\end{array}$ & $\begin{array}{l}\text { Endemic area } \\
\text { Greece }\end{array}$ & $45 / \mathrm{M}$ & PSA & $\begin{array}{l}\text { Methotrexate } \\
\text { Infliximab Prednisolone }\end{array}$ & 60 & $\mathrm{VL}$ & $\begin{array}{l}\text { Pos } \\
\text { BM }\end{array}$ & $\begin{array}{l}\mathrm{Neg} \\
\mathrm{BM}\end{array}$ & $\mathrm{Neg}$ & $\begin{array}{l}\text { Lyophilized } \\
\text { amphotericin }\end{array}$ & Yes & Cured \\
\hline $\begin{array}{l}\text { De Leonardis et al., 2009, } \\
\text { Clin Exp Rheumatol }\end{array}$ & $\begin{array}{l}\text { Endemic area } \\
\text { Italy }\end{array}$ & $63 / \mathrm{M}$ & PSA & $\begin{array}{l}\text { Methotrexate } \\
\text { Infliximab } \\
\text { azathioprine prednisolone }\end{array}$ & 36 & $\mathrm{VL}$ & ND & PosBM & $\mathrm{Neg}$ & $\begin{array}{l}\text { Liposomal amphotericin } \\
\text { B }\end{array}$ & Yes & Cured \\
\hline $\begin{array}{l}\text { Khan et al., 2010, BMJ } \\
\text { case rep }\end{array}$ & $\begin{array}{l}\text { Traveler returning } \\
\text { from Malta }\end{array}$ & $74 / \mathrm{F}$ & PSA & $\begin{array}{l}\text { Adalimumab } \\
\text { methotrexate }\end{array}$ & 21 & $\begin{array}{l}\mathrm{CL} \\
\mathrm{VL}\end{array}$ & $\begin{array}{l}\text { Pos BM } \\
\text { Pos SB }\end{array}$ & $\begin{array}{l}\text { pos BM } \\
\text { pos SB }\end{array}$ & pos & $\begin{array}{l}\text { Intralesional } \\
\text { meglumine antimoniate } \\
\text { and liposomal } \\
\text { Amphotericin }\end{array}$ & Yes & Cured \\
\hline $\begin{array}{l}\text { Romero-Mate et al., 2012, } \\
\text { Dermatol Online J }\end{array}$ & $\begin{array}{l}\text { Endemic area } \\
\text { Spain }\end{array}$ & $59 / \mathrm{F}$ & PSA & $\begin{array}{l}\text { Adalimumab } \\
\text { methotrexate }\end{array}$ & 84 & $\mathrm{CL}$ & Pos & neg & ND & $\begin{array}{l}\text { Intralesional } \\
\text { meglumine antimoniate }\end{array}$ & No & Cured \\
\hline $\begin{array}{l}\text { Besada et al., 2013, } \\
\text { Rheumatol Int }\end{array}$ & $\begin{array}{l}\text { Traveler returning } \\
\text { from } \\
\text { southern Spain }\end{array}$ & $72 / \mathrm{F}$ & PSA & Methotrexate Etanercept & 288 & $\mathrm{VL}$ & Pos BM & Pos BM & ND & $\begin{array}{l}\text { Liposomal amphotericin } \\
\text { B }\end{array}$ & Yes & Cured \\
\hline $\begin{array}{l}\text { Hernandez-Torres et al., } \\
\text { 2013, Scand J Infect Dis }\end{array}$ & $\begin{array}{l}\text { Endemic area } \\
\text { Spain }\end{array}$ & $50 / \mathrm{M}$ & $\mathrm{P}$ & $\begin{array}{l}\text { Corticosteroids } \\
\text { infliximab }\end{array}$ & 36 & $\mathrm{CL}$ & Pos SB & Pos SB & Pos & $\begin{array}{l}\text { Liposomal amphotericin } \\
\text { B }\end{array}$ & Yes & Cured \\
\hline $\begin{array}{l}\text { Guedes-Barbosa et al., } \\
\text { 2013, Semin Arthritis } \\
\text { Rheum (two patients) }\end{array}$ & $\begin{array}{l}\text { Endemic area } \\
\text { Brazil }\end{array}$ & $\begin{array}{l}39 / \mathrm{M} \\
50 / \mathrm{M}\end{array}$ & PSA (2) & $\begin{array}{l}\text { Prednisone } \\
\text { methotrexate adalimumab } \\
\text { (2) }\end{array}$ & $\begin{array}{l}12 \\
14\end{array}$ & $\begin{array}{l}\text { MCL } \\
\mathrm{CL}\end{array}$ & $\begin{array}{l}\mathrm{ND} \\
\mathrm{ND}\end{array}$ & $\begin{array}{l}\text { Pos SB } \\
\text { Pos SB }\end{array}$ & NDND & $\begin{array}{l}\text { Meglumine antimoniate } \\
\text { (2) }\end{array}$ & $\begin{array}{l}\text { No } \\
\text { Yes }\end{array}$ & $\begin{array}{l}\text { Cured } \\
\text { Cured }\end{array}$ \\
\hline $\begin{array}{l}\text { Hadjipetrou et al., 2014, } \\
\text { Infez Med }\end{array}$ & $\begin{array}{l}\text { Endemic area } \\
\text { Greece }\end{array}$ & $65 / \mathrm{M}$ & PSA & $\begin{array}{l}\text { Infliximab }(1) \\
\text { Methotrexate }\end{array}$ & 24 & $\mathrm{VL}$ & Pos BM & Pos BM & Pos & $\begin{array}{l}\text { Liposomal amphotericin } \\
\text { B }\end{array}$ & Yes & Cured \\
\hline $\begin{array}{l}\text { Catala et al., 2014, Actas } \\
\text { Dermosifiliogr }\end{array}$ & $\begin{array}{l}\text { Endemic area } \\
\text { Spain }\end{array}$ & $33 / \mathrm{M}$ & PSA & Infliximab & 24 & $\mathrm{CL}$ & ND & $\begin{array}{l}\text { Pos SB } \\
\text { Neg BM }\end{array}$ & Pos & $\begin{array}{l}\text { Intralesional } \\
\text { meglumine antimoniate }\end{array}$ & Yes & Cured \\
\hline $\begin{array}{l}\text { Rakotonarivo et al., 2018, } \\
\text { JAAD case rep }\end{array}$ & $\begin{array}{l}\text { Traveler returning } \\
\text { from Morocco }\end{array}$ & $62 / \mathrm{M}$ & $\mathrm{P}$ & $\begin{array}{l}\text { Methotrexate } \\
\text { ustekinumab }\end{array}$ & 4 & $\mathrm{CL}$ & Pos SB & Pos SB & ND & $\begin{array}{l}\text { Intralesional } \\
\text { meglumine antimoniate } \\
\text { oral } \\
\text { miltefosine for } 28 \text { days }\end{array}$ & Yes & Cured \\
\hline $\begin{array}{l}\text { Guarneri et al., 2017, } \\
\text { Wien Med Wochenschr }\end{array}$ & $\begin{array}{l}\text { Endemic area } \\
\text { Italy }\end{array}$ & $65 / \mathrm{M}$ & PSA & $\begin{array}{l}\text { Golimumab cyclosporina } \\
\text { metotrexate } \\
\text { steroids }\end{array}$ & 48 & $\mathrm{ML}, \mathrm{VL}$ & ND & $\begin{array}{l}\text { posBM } \\
\text { posSB }\end{array}$ & Pos & $\begin{array}{l}\text { Liposomal amphotericin } \\
\text { B }\end{array}$ & Yes & Cured \\
\hline $\begin{array}{l}\text { Hernandez Bel et al., } \\
\text { 2018, Arch Soc Esp } \\
\text { Oftalmol }\end{array}$ & $\begin{array}{l}\text { Endemic area } \\
\text { Spain }\end{array}$ & $72 / \mathrm{M}$ & PSA & $\begin{array}{l}\text { Prednison } \\
\text { metotrexate } \\
\text { adalimumab }\end{array}$ & 60 & $\mathrm{CL}$ & Pos & Pos & ND & $\begin{array}{l}\text { Intralesional injections } \\
\text { of meglumine } \\
\text { antimoniate }\end{array}$ & Yes & Cured \\
\hline $\begin{array}{l}\text { Martinez-Domenech et } \\
\text { al., 2019, Br J Dermatol } \\
\text { (four patients) }\end{array}$ & $\begin{array}{l}\text { Endemic area } \\
\text { Spain }\end{array}$ & $\begin{array}{l}1 \text { pt } 34 / \mathrm{F} \\
1 \mathrm{pt} 40 / \mathrm{F} \\
1 \mathrm{pt} 71 / \mathrm{M} \\
1 \mathrm{pt} 53 / \mathrm{M}\end{array}$ & $\begin{array}{l}\mathrm{P}(3) \\
\mathrm{PSA}(1)\end{array}$ & $\begin{array}{l}\text { Adalimumab (4) } \\
\text { Etanercept (2) } \\
\text { Metotrexate (2) }\end{array}$ & $\begin{array}{l}17 \\
96 \\
2 \\
24\end{array}$ & $\mathrm{CL}(4)$ & $\begin{array}{l}\text { ND (1) } \\
\operatorname{pos} \mathrm{SB}(3)\end{array}$ & $\begin{array}{l}\text { pos SB(2) } \\
\text { neg SB (2) }\end{array}$ & $\operatorname{neg}(4)$ & $\begin{array}{l}\text { Intralesional injections } \\
\text { of meglumine } \\
\text { antimoniate (4) }\end{array}$ & $\begin{array}{l}\text { Yes (3) } \\
\text { No (1) }\end{array}$ & $\begin{array}{l}\text { Cured } \\
(4)\end{array}$ \\
\hline $\begin{array}{l}\text { Caroleo et al., 2019, Curr } \\
\text { Drug Saf }\end{array}$ & $\begin{array}{l}\text { Endemic area } \\
\text { Italy }\end{array}$ & $48 / \mathrm{M}$ & PSA & $\begin{array}{l}\text { Prednisone } \\
\text { Metotrexate } \\
\text { cyclosporine Adalimumab }\end{array}$ & 12 & $\mathrm{CL}$ & ND & Pos SB & $\mathrm{ND}$ & $\begin{array}{l}\text { Intralesional injections } \\
\text { of meglumine } \\
\text { antimoniate }\end{array}$ & Yes & Cured \\
\hline Present case & $\begin{array}{l}\text { Endemic area } \\
\text { Italy }\end{array}$ & $46 / \mathrm{M}$ & $P$ & Methotrexate & 180 & $\mathrm{VL}, \mathrm{MCL}$ & $\begin{array}{l}\text { pos SB pos } \\
\text { PB }\end{array}$ & ND & Pos & $\begin{array}{l}\text { Liposomal amphotericin } \\
\text { B }\end{array}$ & Yes & Cured \\
\hline
\end{tabular}


rant hyper-proliferation of keratinocytes. The pathogenesis of psoriasis is complex. Genetic susceptibility, environmental triggering factors and an over-reaction of local innate immune response initiate inflammation. Subsequent involvement of adaptive immune response with production of $\mathrm{Th}^{1}$ cytokines, chemokines and growth factors lead to epidermal hyperplasia. ${ }^{5}$

Recently, a functional role of interleukin-17-producing $\mathrm{T}$ helper cells (Th17) in psoriasis has been suggested by their reduction during successful anti-TNF treatment. ${ }^{6}$ It is also known that Th17 lymphocytes play an essential role in protecting against intracellular protozoa and in the successful clearance of Leishmania by strengthening the Th1 response. ${ }^{7}$ In view of this, it could be argued that psoriasis may represent a protective factor for Leishmania infection. Indeed, in our review we did not found any case of leishmaniasis in psoriatic subjects who were not under immunosuppressive therapies.

Biological agents, which are powerful immunosuppressive drugs, have been more and more used in rheumatic patients and Leishmania infections have been reported among anti-TNF-agents users. ${ }^{8}$ Recently Maritati et al. found higher prevalence of subclinical leishmaniasis in patients with inflammatory rheumatic diseases receiving biological drugs than those treated with other immunosuppressive drugs. ${ }^{9}$ However, leishmaniasis has also been reported in psoriatic patients not receiving biological drugs, as occurred to our patient (Table 1 ).

Diagnosis of $\mathrm{CL}$ in psoriatic patients is challenging, as it mimics many other infections or a flare-up of psoriasis itself that can lead to ineffective and harmful changes of therapy. Immunosuppressive therapies cause atypical manifestations of leishmaniasis with large lesions spread over large cutaneous areas and associated to a possible mucosal involvement. ML by L. infantum is very rare and only sporadically described in patients receiving powerful immunosuppressive therapies or in HIV-coinfected patients. MCL is mostly observed in Latin America where L. braziliensis accounts for most cases, but L. panamensis, L. guyanensis, and L. amazonensis have also been implicated. Only rarely cutaneous lesions extend to areas of skin distant from the mucosa involved, as in our case in which two lesions on the foot and knee were associated with the oral lesion. In the context of impaired immunity, it is also advisable to rule out VL by PCR-Leismania on peripheral blood so as to establish the most appropriate therapy: intralesional or intravenous.

Finally, there is no agreement on appropriate screening for leishmaniasis before immunosuppressive treatments and on the strategy to be followed after the diagnosis of leishmaniasis in rheumatic patients taking immunosuppressive drugs. ${ }^{10}$ Molecular methods are highly sensitive and specific tools for the diagnosis of visceral leishmaniasis and a screening with Leishmania-PCR in immunosuppressed patients living in endemic areas could be useful to identify patients at highest risk of reactivation. ${ }^{9}$

Specific leishmaniasis treatment followed by suspension of the immunosuppressive therapy was adopted by most of the authors. Overall even if the treatment response is not as good as seen in the immunocompetent population, our review reports a good outcome in all cases and patients remained relapse-free without maintenance therapy and despite the ongoing use of immunosuppressive medication.

In conclusion physicians must be alert to the possibility of development of leishmaniasis in immunosuppressed rheumatic patients. Adequate screening for VL should be incorporated into the list of baseline studies to carry out before initiating biologic therapies, at least in endemic areas.

\section{Declaration of Competing Interest}

The authors declare that there is no conflict of interest.

\section{Acknowledgment}

This research did not receive any specific grant from funding agencies in the public, commercial, or not-for-profit sector.

\section{Supplementary materials}

Supplementary material associated with this article can be found, in the online version, at doi:10.1016/j.jinf.2020.01.019.

\section{References}

1. Brunet K, Alanio A, Lortholary O, Rammaert B. Reactivation of dormant/latent fungal infection. J Infect 2018;77:463-8. doi:10.1016/j.jinf.2018.06.016.

2. Saporito L, Giammanco GM, De Grazia S, Colomba C. Visceral leishmaniasis: host-parasite interactions and clinical presentation in the immunocompetent and in the immunocompromised host. Int J Infect Dis 2013;17:e572-6. doi:10. 1016/j.ijid.2012.12.024.

3. Erre GL, Mesina P, Tonelli N, Passiu G. Visceral Leishmaniasis among immunosuppressed patients with rheumatic diseases. Clin Exp Rheumatol 2010;28:590-1.

4. Zanger P, Kotter I, Kremsner PG, Gabrysch S. Tumor necrosis factor alpha antagonist drugs and leishmaniasis in Europe. Clin Microbiol Infect 2012;18:670-6. doi:10.1111/j.1469-0691.2011.03674.x.

5. Grozdev I, Korman N, Tsankov N. Psoriasis as a systemic disease. Clin Dermatol 2014:32:343-50. doi:10.1016/j.clindermatol.2013.11.001.

6. Deng Y, Chang C, Lu Q. The inflammatory response in psoriasis: a comprehensive review. Clin Rev Allergy Immunol 2016;50:377-89. doi:10.1007/ s12016-016-8535-X.

7. Gonçalves-de-Albuquerque SDC, Pessoa-E-Silva R, Trajano-Silva LAM, de Goes TC, de Morais RCS, da C Oliveira CN, et al. The Equivocal role of Th17 cells and neutrophils on immunopathogenesis of leishmaniasis. Front Immunol 2017;8:1437. doi:10.3389/fimmu.2017.01437.

8. Cascio A, Iaria M, Iaria C. Leishmaniasis and biologic therapies for rheumatologic diseases. Semin Arthritis Rheum 2010;40:e3-5. doi:10.1016/j.semarthrit.2009.07. 001.

9. Maritati M, Trentini A, Michel G, Bellini T, Almugadam S, Hanau S, et al. Subclinical Leishmania infection in patients with rheumatic diseases under biological drugs. Infection 2018;46:801-9. doi:10.1007/s15010-018-1189-2.

10. Cascio A, Iaria C. Appropriate screening for leishmaniasis before immunosuppressive treatments. Emerg Infect Dis 2009;15:1706 author reply -7. doi:10.3201/ eid1510.090881

Claudia Colomba*, Laura Saporito, Silvia Bonura Department of Health Promotion, Mother and Child Care, Internal Medicine and Medical Specialties "G. D'Alessandro", University of Palermo, Via Liborio Giuffrè, Palermo, Italy

Giuseppina Campisi

Department of Surgical, Oncological and Stomatological Disciplines, University of Palermo, Via Liborio Giuffrè, Palermo, Italy

Paola Di Carlo Department of Health Promotion, Mother and Child Care, Internal Medicine and Medical Specialties "G. D'Alessandro", University of Palermo, Via Liborio Giuffrè, Palermo, Italy

Vera Panzarella Department of Surgical, Oncological and Stomatological Disciplines, University of Palermo, Via Liborio Giuffrè, Palermo, Italy

Valentina Caputo, Antonio Cascio Department of Health Promotion, Mother and Child Care, Internal Medicine and Medical Specialties "G. D'Alessandro", University of Palermo, Via Liborio Giuffrè, Palermo, Italy

*Corresponding author.

E-mail addresses: claudia.colomba@libero.it (C. Colomba), laura.sapo@tin.it (L. Saporito), giuseppina.campisi@unipa.it (G. Campisi), paola.dicarlo@unipa.it (P. Di Carlo), panzarella@odonto.unipa.it (V. Panzarella), valentina.caputo@unipa.it (V. Caputo), Antonio.cascio03@unipa.it

(A. Cascio) 